\title{
Rhabdomyosarcoma of the Cheek Area: About Two Cases and Review of the Literature
}

\author{
F. Baadi ${ }^{*}$, K Zitouni, C. Gakosso, I. Zouita, D. Basraoui, H. Jalal
}

Radiology Department, Mother and Child Hospital, Mohammed VI Chu, Cadi Ayyad University, Marrakech

DOI: $10.36347 /$ sasjm.2020.v06i10.002

| Received: 25.09.2020 | Accepted: 06.10.2020 | Published: 26.10 .2020

*Corresponding author: F. Baadi

Abstract

Case Report

Rhabdomyosarcoma (RMS) is a malignant soft tissue tumor that grows from striated muscle cells. It is the most common malignant mesenchymal tumor in children and adolescents. Jugal rhabdomyosarcomas or rhabdomyosarcomas of the masticatory space are a very rare group of malignant tumors. The purpose of this study is to determine the contribution of imaging in the early diagnosis, extension assessment, management and post-treatment follow-up of this very rare entity.

Keywords: rhabdomyosarcoma, cheek area, MRI, jugal, masticatory muscles.

Copyright $\odot 2020$ The Author(s): This is an open-access article distributed under the terms of the Creative Commons Attribution 4.0 International License (CC BY-NC 4.0) which permits unrestricted use, distribution, and reproduction in any medium for non-commercial use provided the original author and source are credited.

\section{INTRODUCTION}

Rhabdomyosarcoma (RMS) is a malignant soft tissue tumor that grows from striated muscle cells. It is the most common malignant mesenchymal tumor in children and adolescents.

Jugal rhabdomyosarcomas or rhabdomyosarcomas of the masticatory space are a very rare group of malignant tumors, which are considered to have a good prognosis. They pose a double problem: positive diagnosis and therapeutic management.

Facial MRI is the examination of choice for diagnostic orientation, making a locoregional extension assessment but diagnostic confirmation requires an anatomopathological examination coupled imperatively with an immunohistochemical study.

Furthermore, the difficulty of therapeutic management requires the intervention of a multidisciplinary team in order to improve the prognosis.

\section{PRESENTATION OF CLINICAL CASES}

We report two observations collected in the mother and child radiology department:

\section{Case-1}

A 7-year-old child who presents with a swelling of the right cheek for 4 months without other associated signs. The clinical examination noted a swelling of the right cheek without any inflammatory lesion opposite, prolapsed at the endo buccal level encompassing the Sténon duct.

Ultrasonography revealed a heterogeneous hypoechoic tissue lesion, presence of flow in Doppler color. MRI showed that a well-limited subcutaneous soft tissue mass of the right cheek, in isosignal T1, hyper intense T2, was heterogeneously in enhancement on injected sequence. In the interior, presence contact with the masseter muscle with respect to the fat separation border and the buccinator muscle extended to the alveolar rim where it loses its limit. It encompasses the canal of the ipsilateral Sténon, which it dilated, absence of cervical adenopathy's (Figures 14).

The child benefited from a surgical biopsy, and the anatomopathological study in favor of a rhabdomyosarcoma of the cheek.

With no metastasis locations and the child beneficed on neoadjuvant chemotherapy. 


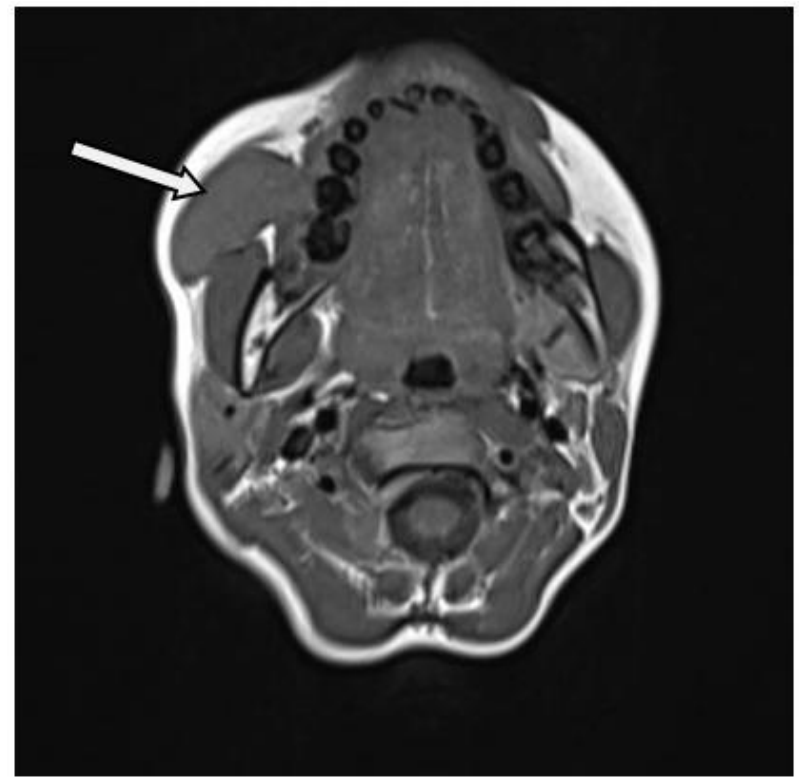

Fig-1: Facial MRI in axial section T1 sequence: Straight jugular tissue formation in hypo signal $\mathrm{T} 1$ sequence

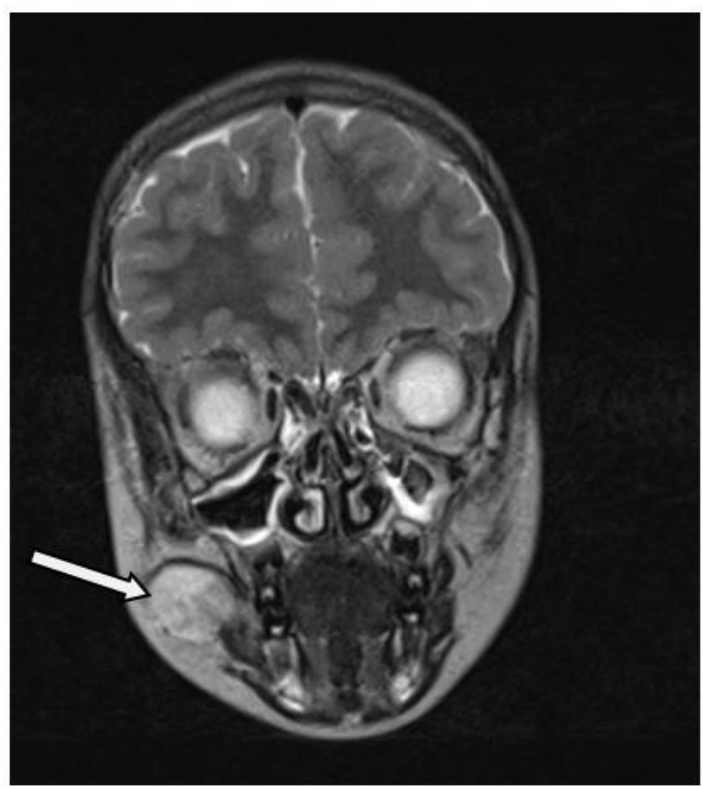

Fig-2: Coronal section facial MRI, T2 sequence: straight jugular tissue formation in heterogeneous $\mathrm{T} 2$ hypersignal

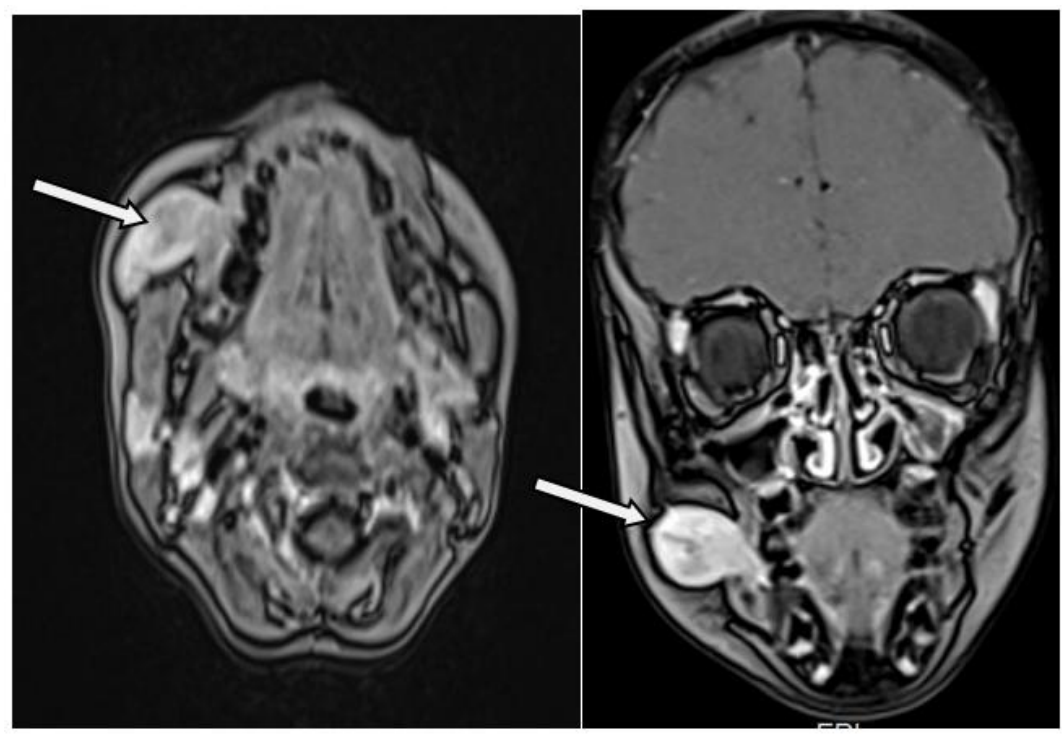

Fig-3: Facial MRI in axial and coronal sections, T1 sequences after gadolinium injection: straight jugular tissue formation rises heterogeneously after gadolinium injection

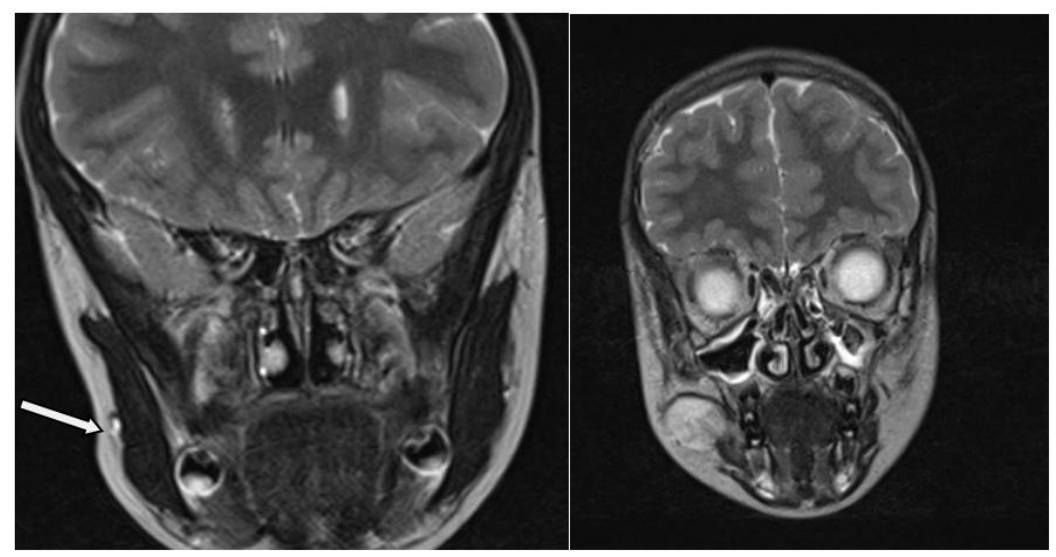

Fig-4: Coronal section facial MRI, T2 sequence: this straight jugular tissue formation encompasses a dilated stenon duct (arrow) 


\section{Case-2}

A 9-yearold child, who presented with a left jugular swelling for 7 months.

US showed a tissue lesion at the expense of the masseter muscle, a heterogeneous hypoechoic muscle well vascularized on Doppler color.

MRI shows a tissue mass of the subcutaneous soft tissues of the left cheek; at the expense of the masseter muscle, well limited, in isosignal T1, hyper intense T2 and Flair; which enhancement heterogeneously in injected sequence (Figures 5-7).

Internally presence the contact with the lateral pterygoid muscle with loss of the fatty separation border, and with the mandibular angle and the lower dental arches, without cervical adenopathy's or visible bone lysis.

The child benefited from a surgical biopsy, and the anatomopathological study in favor of a rhabdomyosarcoma of the cheek.

Without metastasis lesions, the child received chemotherapy with a post-therapy with control MRI.

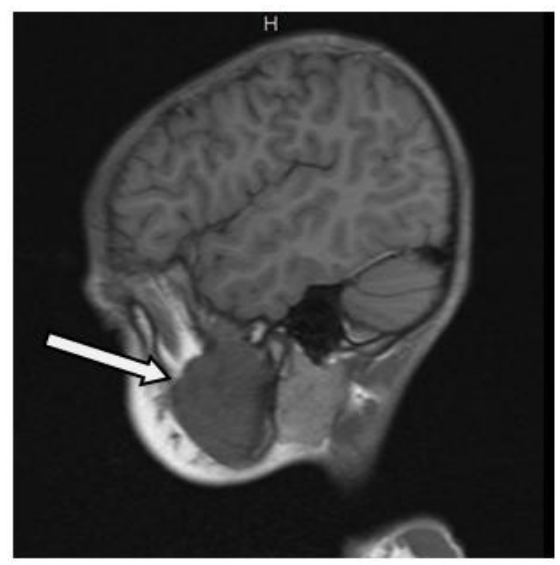

Fig-5: Facial MRI in sagittal section, sequence T1: left jugular tissue formation depending on the muscle masseter iso signal T1

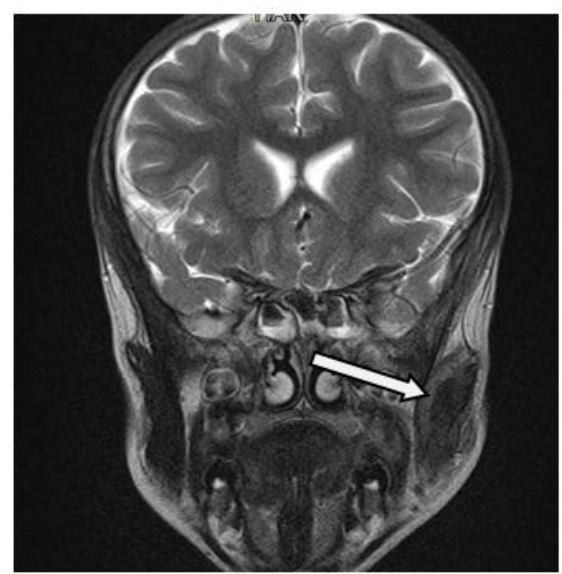

Fig-6: Coronal section facial MRI, T2 sequence: left jugular tissue formation depending on the masseter muscle, heterogeneous $\mathrm{T} 2$ signal

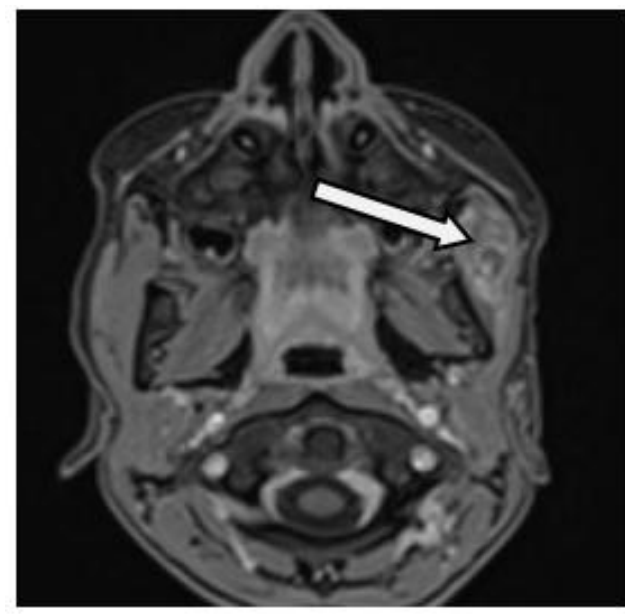

Fig-7: Facial MRI in axial section, T1 sequence after gadolinium injection: left jugular tissue formation depending on the masseter muscle heterogeneously enhanced after gadolinium injection

\section{DISCUSSION}

Malignant mesenchymal tumors in children account for $5-10 \%$ of all malignant tumors in children. They constitute a heterogeneous group of tumors with varied differentiation according to their presumed tissue of origin.

They mainly include tumors of muscular origin, those derived from connective, vascular, nervous or adipose tissue [1].

Rhabdomyosarcoma (RMS) is the most common malignant mesenchymal tumor in children and adolescents $(60-70 \%)$. It is a tumor with striated muscle differentiation.

The annual incidence of RMS in children is 4.3 cases per 1 million children. Two thirds of RMS diagnosed in children under 6 years of age with another peak in adolescence [2], which is the case with our two observations. It can occur anywhere in the body, including sites where striated muscle tissue is not normally present.

The most frequent sites are the head and neck (40\%), where orbital localization is the most common, the other localizations are : the oral cavity, oropharynx, larynx, sinus, nasal fossa, parotid and palate [3], the jugal or masticatory space localization is exceptional; the genitourinary tract $(20 \%)$, including para-testicular or uterovaginal tumors, limbs $(20 \%)$ and trunk $(20 \%)$ [4].

The initial clinical semiology of rhabdomyosarcomas of the cheek is poor, not very suggestive of the real nature of the lesion concerned, and generally boils down to the existence of a more or less painful cheek mass discovered by the parents or the child himself, as in our cases. 
There are few clinical characteristics, which can point to a malignant pathology. The age of the child and the location of the mass can help; but cannot be retained as sufficiently sensitive criteria of benignity or malignity as shown in our two clinical presentations.

A height greater than $5 \mathrm{~cm}$, usually described as associated with malignancy especially in adults, was not a significant endpoint in our study [5].

RMS is a locally infiltrating tumor, invading neighboring organs and destroying bone by contiguity (particularly in the head and neck), but the lesion remains very limited in two presentations, with no extensive bone lysis or involvement of deep facial spaces [6].

Imaging plays a key role in positive diagnosis by specifying the exact site and size of the lesion, in the assessment of local extension to the neighboring and intracranial compartment and at a distance, and in posttherapy follow-up.

The first examination carried out is an ultrasound of the cheek and cervical region, using a superficial probe with color Doppler study, which allows the lesion to be highlighted; specifying its solid, cystic, mixed nature, study its vascularization. Its local extension and it also looks for cervical adenopathy's, but the study is hampered by bone and air so its study in deep spaces is limited.

Although the study of the cheek lesion and its local extension is specified mainly by MRI, because of its excellent contrast resolution and non-irradiating nature. It also allows a study on several levels, giving the surgeon a complete perspective of the tumor's contacts with neighboring structures, enabling him to prepare and anticipate the surgical procedures, necessary to perform a satisfactory carcinological exeresis even before the per-operative assessment.

Whole-body MRI to search for distant metastases can be performed [7]. The disadvantages of this technique are the very long examination time (30 to $45 \mathrm{~min}$ ), sensitivity to movement artifacts and most children require sedation or general anesthesia during the examination [8].

The routine protocols are the axial $\mathrm{T} 1$ sequences, the T1 FATSAT sequences without in with injection of contrast media, in the three plans (3D): sequences axial, sagittal and coronal. The axial and coronal T2 sequences, the FLAIR and T2* sequence for the study of endocranial extension.

The diffusion sequence is an essential sequence for orientation towards malignant lesions, the search for lymph node involvement and the differentiation between a residual lesion and posttherapeutic remodeling [9].

Most lesions are single, localized lesions although multifocal primary RMS has been reported [10]. The T1-W spin echo sequences provide excellent contrast between the tumor and the surrounding adipose planes, as well as the base of the skull in the axial and coronal planes.

The T2 sequences can show a wide variety of RMS signal in different patients: hypo intense, hyper intense or intermediate signal, relative to muscle can be seen $[11,12]$. Some tumors may appear relatively homogeneous and others are frankly heterogeneous, probably correlated with tumor necrosis and tumor cell matrix [13, 14].

Generally, there is no pathognomonic aspect on MRI of rhabdomyosarcomas of the masticatory space and the presence of a lesion in this space poses the diagnostic problem with other tumor and pseudotumor pathology including lymphoma, tumors of the salivary glands, tumor of the Sténon duct, tuberculosis, Sténon duct lithiasis and lipoma.

CT allows a very rapid examination with a better study of the bone with a multi-planar study, but the use of $\mathrm{CT}$ is likely to induce radio-induced cancer [15].

The diagnosis is always must be confirmed histologically. Fine Needle aspiration is often easy but has the disadvantage of limiting the tumor tissue available for histological and immuno-histochemical examination.

At surgical biopsy, the fragment should be of sufficient size to allow cytogenetic or molecular biology studies [6]. There are two main histological forms: the embryonic type with two subtypes of good prognosis (the botryoid and spindle cell type) and the alveolar type [16]. At the head and neck level, alveolar histology is the most frequent [17].

Lymphatic extension is most common in limb tumors. The most frequent metastatic sites are the lungs but also to bone, bone marrow and subcutaneous tissue, hence an exhaustive extention assessment is required including a biological assessment and a thoracoabdominal CT scan, Liver ultrasound, bone scan, fluorodeoxyglucose positron emission tomography (FDG-PET) [18], bone marrow biopsy and possibly lumbar puncture [19].

The therapeutic indications vary according to the extension assessment. As rhabdomyosarcomas are particularly chemo sensitive, it generally combines surgery with chemotherapy for localized tumors as in our cases. The course depends essentially on the 
localization, local and distant extension, the age of the child and the histological type of the tumor (embryonic or alveolar).

\section{CONCLUSION}

Rhabdomyosarcoma is most common in school-aged children, and less common in older people. The diagnosis and treatment of this pathology must be early, as the prognosis for less advanced forms is favorable. This diagnosis should always be evoked in the presence of any rapidly progressive jugal mass in child hood, but also in adults.

\section{REFERENCES}

1. Bamaarouf S. Les rhabdomyosarcomes de l'enfant: Experience $d u$ service d'oncologie pediatrique de l'hopital d'enfant de rabat (Doctoral dissertation). Thèse de Médecine de Rabat $\mathrm{N}^{\circ} 73 / 2008$.

2. Pizzo, Philip A, Poplack, David G. Rhabdomyosarcoma and the undifferentieted sarcoma. Principles \& Practice of Pediatric Oncology, 5th Edition, 2006: 971-996.

3. Tazi M, Hafian H, Collin F, Maigon P, Bruchon Y, Perrin D. Rhabdomyosarcome du maxillaire: à propos d'une observation. Médecine Buccale Chirurgie Buccale. 2002;8(2):81-6.

4. Flamant F, Rodary C, Rey A, Praquin MT, Sommelet D, Quintana E, Theobald S, BrunatMentigny M, Otten J, Voute PA, Habrand JL. Treatment of non-metastatic rhabdomyosarcomas in childhood and adolescence. Results of the second study of the International Society of Paediatric Oncology: MMT84. European journal of cancer. 1998 Jun 1;34(7):1050-62.

5. van Rijswijk CS, Geirnaerdt MJ, Hogendoorn PC, Taminiau AH, van Coevorden F, Zwinderman AH, Pope TL, Bloem JL. Soft-tissue tumors: value of static and dynamic gadopentetate dimeglumineenhanced MR imaging in prediction of malignancy. Radiology. 2004 Nov;233(2):493502.

6. Coulibaly $\mathrm{AD}$. Aspects épidémiologique clinique et thérapeutique du rhabdomyosarcome chez l'enfant dans le département de Pédiatrie au Centre Hospitalier Universitaire Gabriel Touré. Thèse de Médecine de Bamako. 2018.

7. Canale S, Lemery M, Vilcot L, Ammari S, Bidault F, Balleyguier C, Caramella C, Dromain C. Service, Place de l'IRM corps entier dansl'évaluation des tumeurs de l'enfant, Journal de Radiologie Diagnostique et Interventionnelle. 2014.

8. Goo HW, Choi SH, Ghim T, Moon HN, Seo JJ. Whole-body MRI of paediatric malignant tumours: comparison with conventional oncological imaging methods. Pediatric radiology. 2005 Aug 1;35(8):766-73.

9. Razek AA, Megahed AS, Denewer A, Motamed A, Tawfik A, Nada N. Role of diffusion-weighted magnetic resonance imaging in differentiation between the viable and necrotic parts of head and neck tumors. Acta Radiologica. 2008 Jan 1;49(3):364-70.

10. Sohaib SA, Moseley I, Wright JE. Orbital rhabdomyosarcoma-the radiological characteristics. Clin Radiol. 1998; 53:357-362

11. Kim EE, Valenzuela RF, Kumar AJ, Raney RB, Eftekari F. Imaging and clinical spectrum of rhabdomyosarcoma in children. Clinical imaging. 2000 Sep 1;24(5):257-62.

12. Yousem DM, Lexa FJ, Bilaniuk LT, Zimmerman RI. Rhabdomyosarcomas in the head and neck: MR imaging evaluation. Radiology. 1990 Dec;177(3):683-6.

13. Srinivasan A, Dvorak R, Perni K, Rohrer S, Mukherji SK. Differentiation of benign and malignant pathology in the head and neck using 3T apparent diffusion coefficient values: early experience. American journal of neuroradiology. 2008 Jan 1;29(1):40-4.

14. Sakamoto J, Yoshino N, Okochi K, Imaizumi A, Tetsumura A, Kurohara K, Kurabayashi T. Tissue characterization of head and neck lesions using diffusion-weighted MR imaging with SPLICE. European journal of radiology. 2009 Feb $1 ; 69(2): 260-8$.

15. Brenner DJ, Elliston CD, Hall EJ, Berdon WE. Estimated risks of radiation-induced fatal cancer from pediatric CT. American journal of roentgenology. $2001 \mathrm{Feb}$;176(2):289-96.

16. Leila $H$. Etude du rhabdomyosarcome chez l'enfant dans le service d'oncologie et hématologie pédiatrique, Thèse de Médecine de Marrakech N $171 / 2017$

17. Little DJ, Ballo MT, Zagars GK, Pisters PW, Patel SR, El-Naggar AK, Benjamin RS. Adult rhabdomyosarcoma. Cancer. 2002; 95(2): 377 388.

18. Bastiaannet E, Groen H, Jager PL, Cobben DCP, Van Der Graaf WTA, Vaalburg W, Hoekstra HJ. The value of FDG-PET in the detection, grading and response to therapy of soft tissue and bone sarcomas; a systematic review and meta-analysis. Cancer treatment reviews. 2004; 30(1): 83-101.

19. Arrob A, Fiqhi MK, N'diaye A, El Khatib K, Abouchadi A. Rhabdomyosarcome cranio-facial de l'adulte: un cas de mauvais pronostic. The Pan African Medical Journal. 2018;30. 